\title{
CRISPR interference of nucleotide biosynthesis improves production of a single-domain antibody
}

\author{
Jenny Landberg ${ }^{1}$, Naia Wright ${ }^{1}$, Tune Wulff ${ }^{1}$, Markus Herrgard ${ }^{1,1}$, and Alex Nielsen ${ }^{1}$ \\ ${ }^{1}$ Technical University of Denmark
}

May 27, 2020

\begin{abstract}
Growth decoupling can be used to optimize production of biochemicals and proteins in cell factories. Inhibition of excess biomass formation allows for carbon to be utilized efficiently for product formation instead of growth, resulting in increased product yields and titers. Here, we used CRISPR interference (CRISPRi) to increase production of a single domain antibody (sdAb) by inhibiting growth during production. First, we screened 21 sgRNA targets in the purine and pyrimidine biosynthesis pathways, and found that repression of 11 pathway genes led to increased GFP production and decreased growth. The sgRNA targets pyrF, pyrG, and cmk were selected and further used to improve production of two versions of an expression-optimized sdAb. Proteomics analysis of the sdAb-producing pyrF, pyrG, and cmk growth decoupling strains showed significantly decreased RpoS levels and an increase of ribosome-associated proteins, indicating that the growth decoupling strains do not enter stationary phase and maintain their capacity for protein synthesis upon growth inhibition. Finally, sdAb production was scaled up to shake-flask fermentation where the product yield was improved 2.6-fold compared to the control strain with no sgRNA target sequence. An sdAb content of $14.6 \%$ was reached in the best-performing pyrG growth decoupling strain.
\end{abstract}

\section{Hosted file}

CRISPR interference of nucleotide biosynthesis improves production of a single domain antibody.pdf available at https://authorea.com/users/326703/articles/454516-crispr-interference-ofnucleotide-biosynthesis-improves-production-of-a-single-domain-antibody 


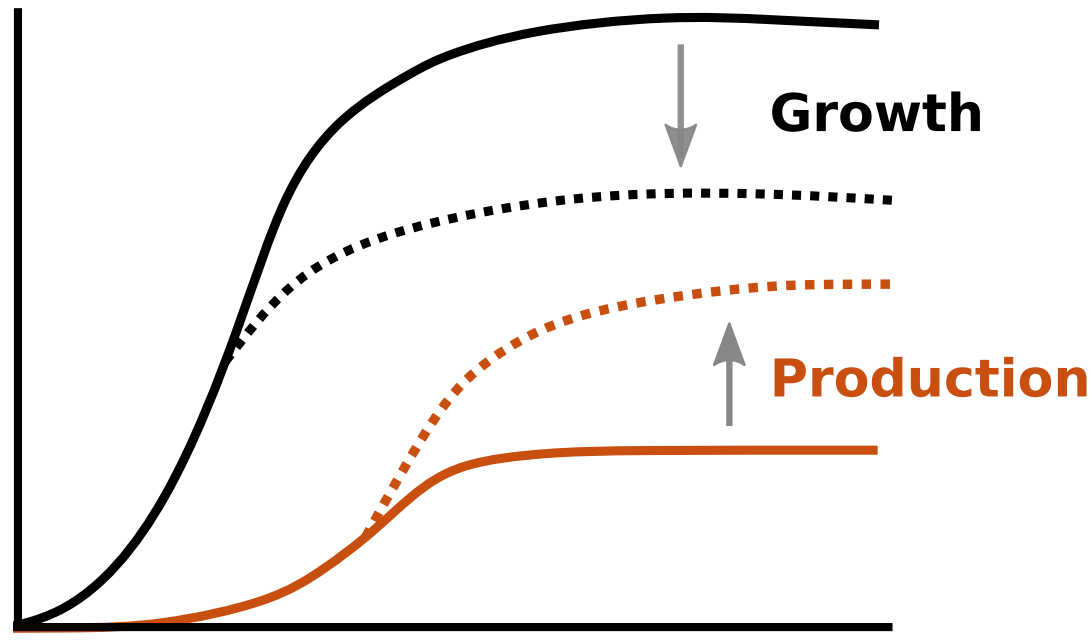

- Regular growth and production ..... Growth decoupling 

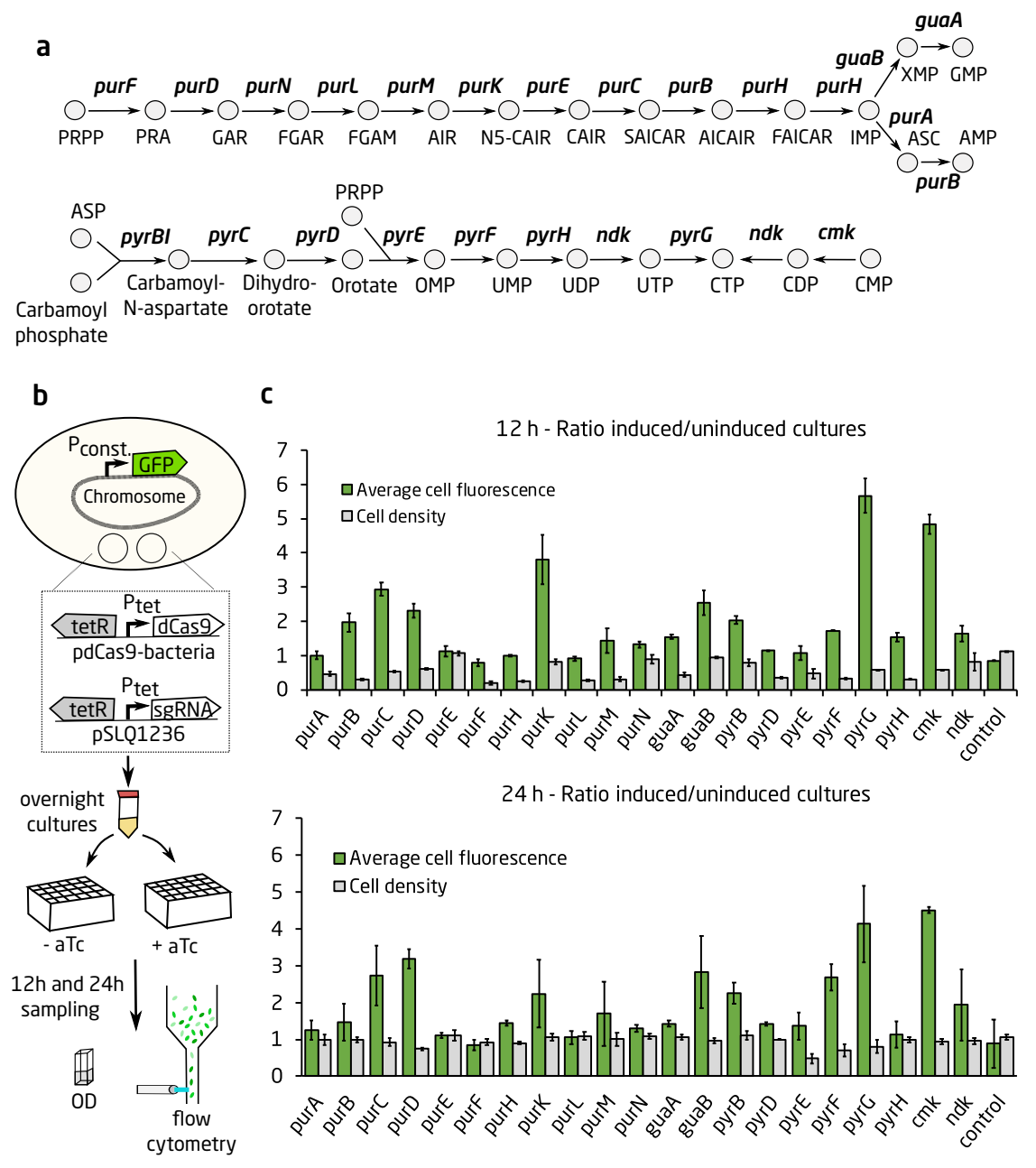

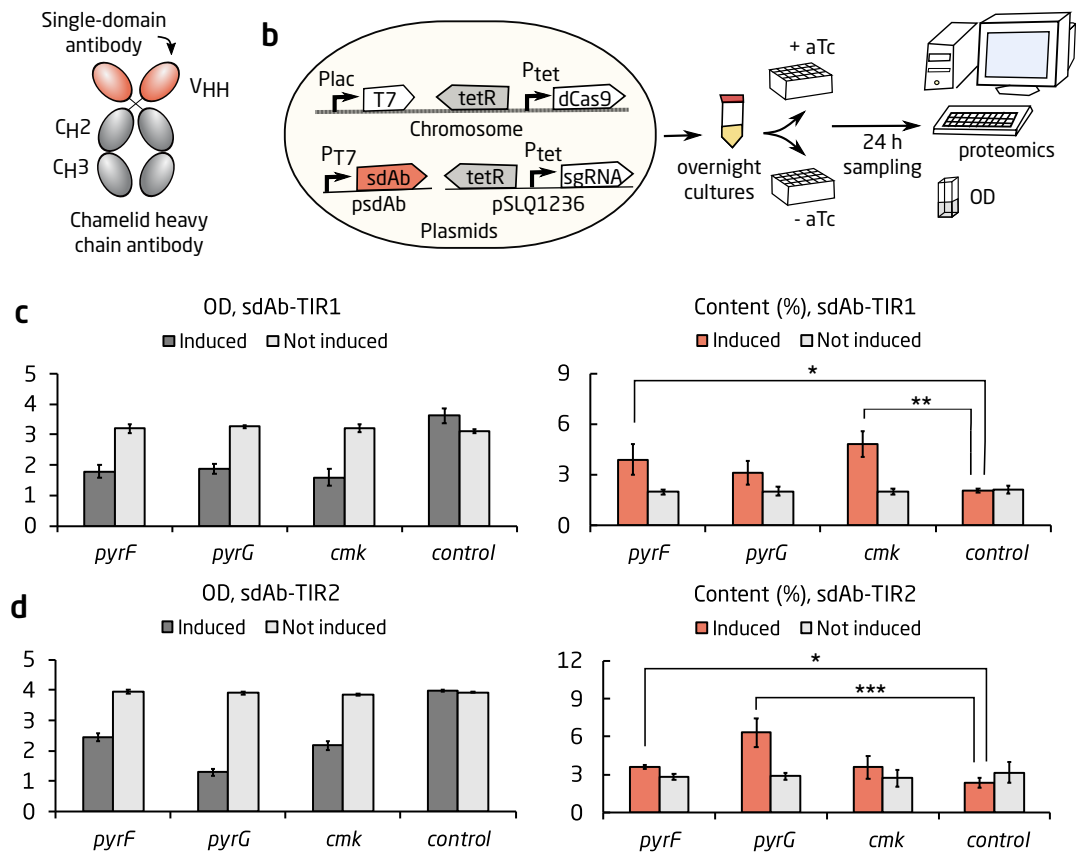

pyrf pyrf pyrG pyrG cmk cmk TIR1 TIR2 TIR1 TIR2 TIR1 TIR2

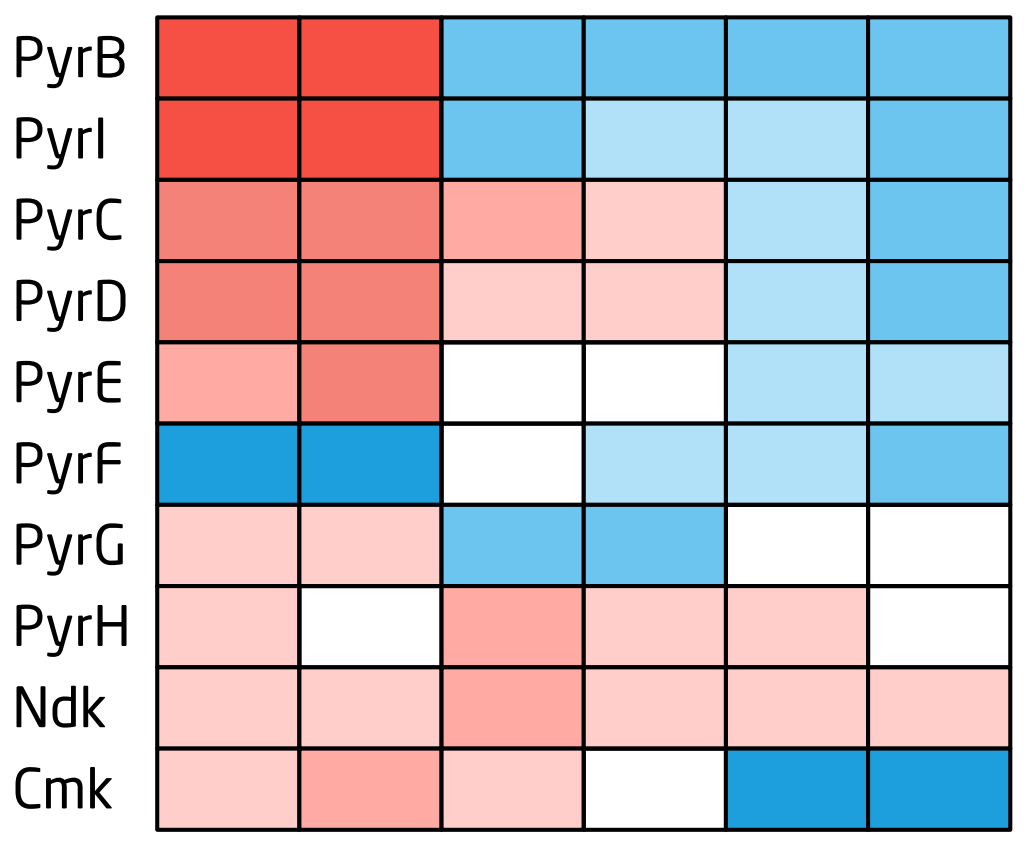

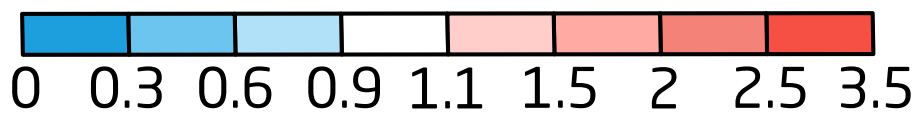




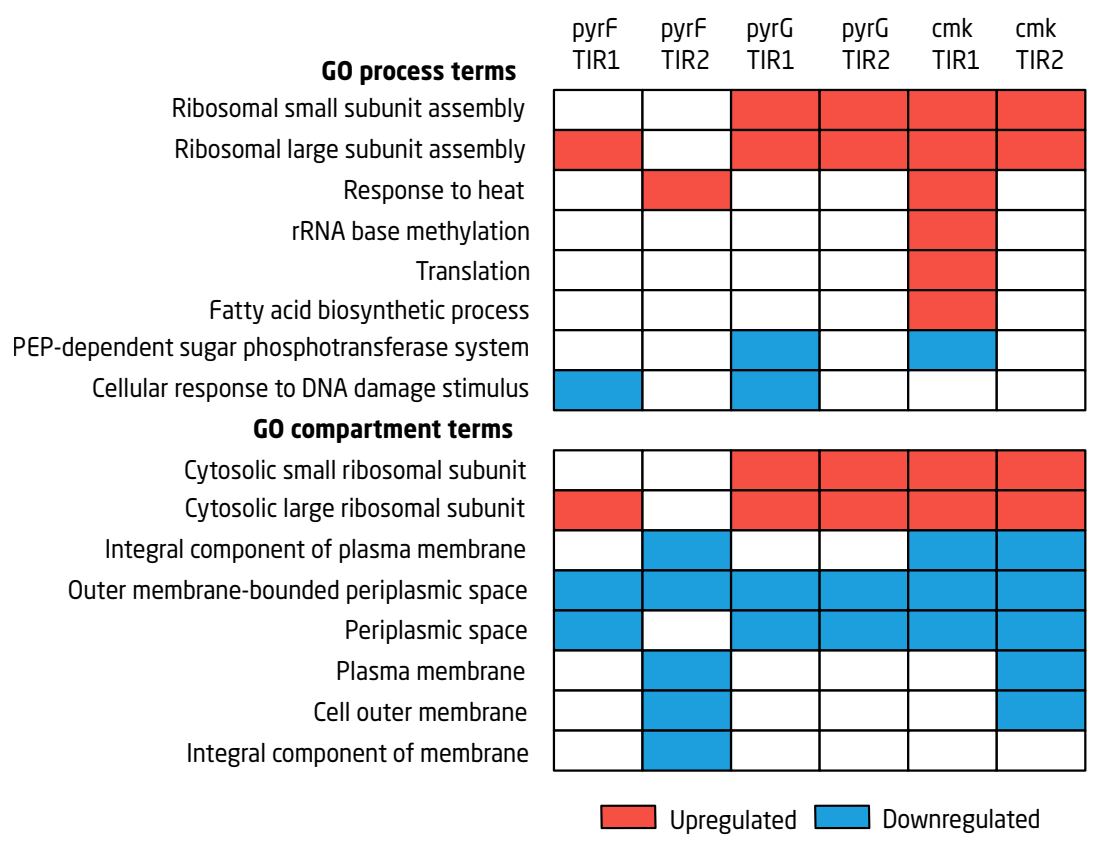

a

OD, sdAb-TIR1 Content (\%), sdAbTIR1

b

OD, SDAD-TIR2

Content (\%), sdAbTIR2

$\square$ Induced $\square$ Not induced

口Induced $\square$ Not induced

口Induced $\square$ Not induced

口Induced $\square$ Not induced
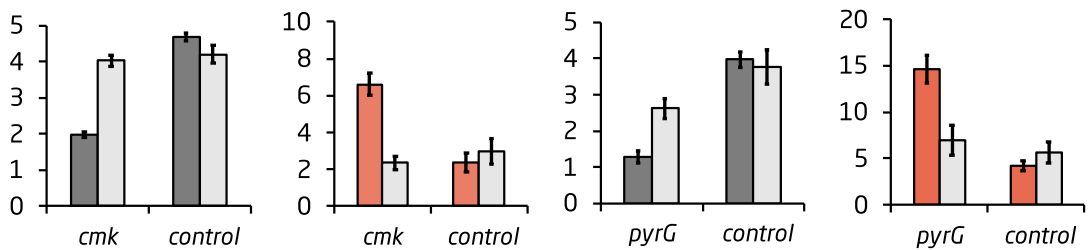